\title{
Improving the Bit Error Rate of OFDM using Convolutional codes
}

\author{
R. Prakash Kumar \\ CVR College of Engineering/E.C.E Department, Hyderabad, India \\ Email: prakash.rachmagdu@gmail.com
}

\begin{abstract}
In wireless applications high throughput and better transmission quality can be achieved by parallel transmission of data, one of such technique is Orthogonal Frequency Division Multiplexing (OFDM) by adding channel coding to the uncoded OFDM ,the performance can be improved. In this paper, the system throughput of a working OFDM system has been enhanced by adding convolution coding. Convolution codes are used extensively in numerous applications in order to achieve reliable data transfer, including digital audio and video, mobile, radio communication, satellite deep space network communication. They can correct even burst and random errors. Simulation is done over Additive white Gaussian Noise (AWGN) channel has 64- sub carriers each is individually modulated by 16-QAM (Quadrature Amplitude Modulation). The performance parameter used for evaluation of BER(Bit Error Rate) with AWGN channel.
\end{abstract}

Index Terms-OFDM, BER, Convolution codes.

\section{INTRODUCTION}

The FEC (Forward Error Correction) have become a vital part of modern digital wireless communication systems enabling reliable transmission to be achieved by over noisy channel by adding the redundancy to the transmitted data. These additional bits (redundancy), while conveying no new information themselves, make it possible for channel decoder to detect and correct information bearing bits. Error detection and correction lowers the overall probability of error. Over the past years convolutional codes have been widely considered to be most practical importance. Convolutional code maps information blocks of length ' $\mathrm{k}$ ' to code blocks of length ' $n$ '. This linear mapping contains memory, because the encode block depends on ' $m$ ' previous information blocks. In IEEE $802.11 \mathrm{a} / \mathrm{g}$ transmitters, convolution codes are used for FEC at the physical layer.

\section{SYSTEM MODELING}

The block diagram of convolution coded OFDM shown in Fig. 1. It consists of transmitter, AWGN channel and receiver. The transmitter consists of Convolution encoder and OFDM modulator. The information bits are encoded by convolution encoder, converts serial data into parallel data. This information is modulated by a 16-QAM modulator mapping onto the sub carrier amplitude and phase, and then transforms this spectral representation of the data into the time domain using an Inverse Fast Fourier Transform (IFFT). During modulation, OFDM symbols are divided into frames such that data will be modulated by frame in order to receive signal be in SYNC with the receiver. In order to eliminate ISI (Inter Symbol Interference) and ICI (Inter Carrier Interference) a guard time (cyclic prefix) is inserted between the OFDM symbols. This allows the receiver to capture the starting point of a symbol period, such that FFT (Fast Fourier Transform) has corrected the information bits.

This signal is passed through an AWGN channel. The important property of this channel is it is a universal channel and linear in all frequencies of amplitude and phase, so 16- QAM modulated signal passes through it without any loss of amplitude and phase. Apart from this fading doesn't exit. There by received signal is the summation of original signal and white Gaussian noise.

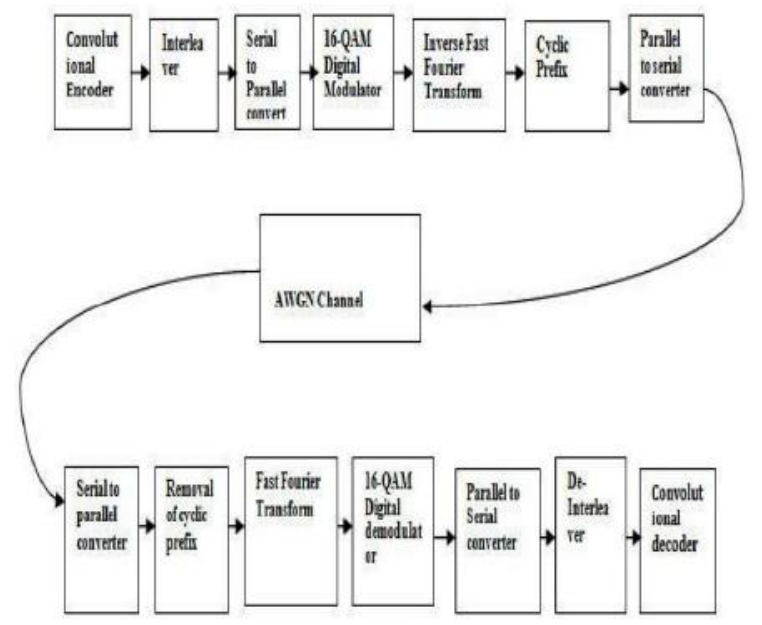

Figure 1 Block diagram of convolution coded OFDM

The receiver performs the inverse operation of transmitter. First, the CCOFDM (convolution coded OFDM) data are split from a serial stream into parallel sets. The Fast Fourier Transform (FFT) converts the time domain samples back into a frequency domain 
representation. The magnitudes of the frequency components correspond to the original data demodulated by 16-QAM demodulator. Finally, the parallel to serial block converts this parallel data into a serial stream to recover the original input data is decoded by viterbi algorithm.

\section{CONVOLUTION ENCODER}

An $(\mathrm{n}, \mathrm{k}, \mathrm{m})$ convolutional code can be implemented with a k-input, n-output linear sequential circuit with input memory $\mathrm{m}$.it encodes the data by passing through a linear finite state-shift register. Depends on the shift register connection encoders are two types, one is feed forward and other is feedback systematic and Nonsystematic. In this paper the Encoding process begins with the generation of 600000 random binary bits. For text data type, the first step is decimal to binary conversion. Then it is encoded by convolutional code of rate $1 / 2$. With the constraint length of 7 and feedback connections are (133) and (171) with generator upper polynomial matrix, [1 111 1 1 and under polynomial matrix, $\left[\begin{array}{lll}1 & 0 & 1\end{array}\right]$. The output of this block is codeword with length 1200000 bits. Through 16- QAM block codeword is mapped into signal with the possibility of phase and amplitude formed. 16-QAM modulator produces symbols in which each symbol represents 4 bits.

\section{OFDM MODULATOR}

The main principle of OFDM is the high-speed distribution of a data stream into a number of low-speed data streams, then, sent through subcarrier simultaneously. Row of data is converted into information in the form of parallel. The original bit rate $\mathrm{R}$ is transmitted into a parallel path $\mathrm{R} / \mathrm{N}$, where $\mathrm{N}$ refers to number of 64-subcarriers (equal to the number of parallel paths) of which each datum is modulated by subcarrier with FFT size 64 using the IFFT to form OFDM symbols. Equation (1) [5] shows the IFFT process that allows the allocation of OFDM symbols in the form of time. The output of the IFFT OFDM symbols forms a mutually orthogonal in time domain.

$x(n)=\sum_{n=0}^{N-1} x(k) \sin \left(\frac{\pi k n}{\mathbb{N}}\right)-i \sum_{n=0}^{N-1} x(k) \cos \left(\frac{2 \pi k n}{N}\right)$

Where $x(n)$ is the IFFT output signal, $x(k)$ is the transmitted signal at the $\mathrm{k}^{\text {th }}$ and $\mathrm{N}$ is the number of subcarriers. OFDM symbol is formed after the next process is added with cyclic prefix $25 \%$ of the total subcarrier channel that serves as a guard to avoid ISI. After the addition of the cyclic prefix represented in Equation 2, the data are converted back to serial form that can be transmitted from the channel parallel to serial converter.

$$
\mathrm{T}_{\text {total }}=\mathrm{T}+\mathrm{T}_{\mathrm{g}}
$$

Where $\mathrm{T}$ is the OFDM symbol length without cyclic prefix, $T_{g}$ is the length of cyclic prefix and the OFDM symbol $\mathrm{T}_{\text {total }}$ is the overall length.

\section{OFDM DEMODULATOR}

OFDM decomposes a signal using Fast Fourier Transform (FFT). Its function is convert time domain signal into frequency domain. The FFT process can be represented by (3) [5]. This process is also done in the disposal of cyclic prefix.

$$
x(k)=\sum_{\mathrm{n}=1}^{N-1} x(\mathrm{n}) \sin \left(\frac{2 \pi k n}{\mathrm{~N}}\right)+\mathrm{i} \sum_{\mathrm{n}=0}^{\mathrm{N}-1} x(\mathrm{n}) \cos \left(\frac{2 \pi k n}{\mathrm{~N}}\right)
$$

Where $\mathrm{x}(\mathrm{n})$ is the signal at time $\mathrm{n}$; $\mathrm{N}$ is the number of subcarriers (subcarrier $=64$ ), $\mathrm{k}$ is the frequency index of $\mathrm{N} ; \mathrm{n}$ is the index of time that produces $\mathrm{x}(\mathrm{k})$ that is the value of the frequency spectrum at $\mathrm{k}$.

\section{CONVOLUTION DECODER}

There are two basic categories to decoding convolutional codes. They are sequential decoding and maximum likelihood decoding based on Fano algorithm and Viterbi algorithm respectively. In this paper we are using the Viterbi decoding is an efficient and practical decoding for short constraint lengths. The following procedure used to trellis encoded data decoded by viterbi algorithm. Decoder is start with initialization of resetting all registers to 0's hamming distance has been calculated The Vitrerbi Decoder is a "maximum likelihood" decoder; this means that every time an input symbol is identified as "invalid" (i.e. it cannot be the direct output of a specified counvolutional encoder, no matter what sequence has been fed into the encoder), the decoder will try to assume the most likely symbol to have been transmitted by the encoder. The likelihood criteria are established based on a number of assumptions on the characteristics of the transmission channel. The Viterbi Decoder assumes that the transmission channel is memory less (i.e. its characteristics at a given moment do not depend on the previous channel state), the transfer function of the channel is linear (a non-linear function would produce cross-modulation effects), the transfer function is timeinvariant, and a Gaussian additive noise is present on the channel.

\section{SIMULATION RESULTS}

The main goal of this paper was to simulate OFDM system by utilizing convolution coding in Matlab. The CCOFDM simulated parameter and their values are shown in Table 1.in this paper CCOFDM generates total number of frames are 100, each frame consists of 96bits. Each frame is modulated by 16-QAMand 64subcarrier OFDM system with convolution code rate $1 / 2$ and feedback connections are (133) and (171) is 
simulated. Simulation algorithm steps and parameters as shown in Table 1.

TABLE I

THE CCOFDM SIMULATION PARAMETERS

\begin{tabular}{|l|l|}
\hline Simulation parameter & value \\
\hline Number of subcarriers & 64 \\
\hline Convolution code & $1 / 2(133,171)$ \\
\hline IFFT size & 64 \\
\hline Modulation scheme & $16-\mathrm{QAM}$ \\
\hline SNR & $(0-16) \mathrm{dB}$ \\
\hline Single Frame Size & $96-$ bits \\
\hline $\begin{array}{l}\text { Total number of } \\
\text { Frames }\end{array}$ & 100 \\
\hline Number of pilots & 4 \\
\hline Cyclic extension & $25 \%(16)$ \\
\hline Channel & AWGN \\
\hline
\end{tabular}

Simulation of Algorithm steps:

Here, we measured the performance of the CCOFDM through MATLAB simulation. The simulation follows the procedure listed below:

Step 1: 9600 information bits are randomly generated by built in command is randint $(9600,1)$.

Step 2: Encode the information bits using a convolutional encoder with the specified $(133,171)$ poly2trellis matrix.

Step 3: 16-QAM modulation convert the binary bits, 0 and 1, into complex signals (before these modulations use zero padding) .each signal is 4-bits.

Step 4: Performed serial to parallel conversion.

Step 5: Use IFFT to generate OFDM signals, total 100 frames are generated each frame size of 96-bits.

Step 6: Use parallel to serial convertor to transmit signal serially.

Step 7: Introduce noise to simulate channel errors. We assume that the signals are transmitted over an AWGN channel. The noise is modeled as a Gaussian random variable with zero mean and variance the variance of the noise is obtained by a probability function as

$$
f(x)=\frac{1}{\sigma \sqrt{2 \pi}} e^{\frac{(x-m) 2}{2 \pi 2}}
$$

Step 8: At the receiver side, perform reverse operations to decode the received sequence.

Step 9: Count the number of erroneous bits by comparing the decoded bit sequence with the original one.

Step 10: Calculate the BER and plot it.

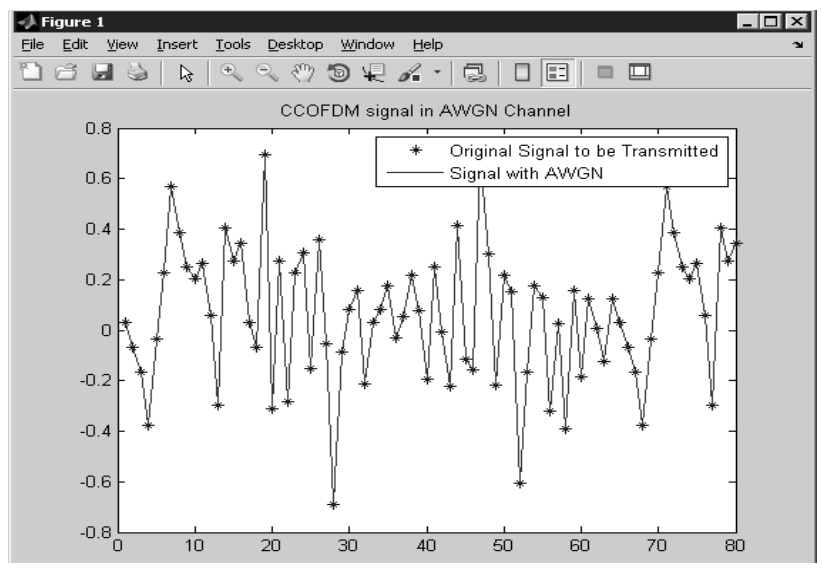

Figure 2. CCOFDM signal in the AWGN channel

Three methods are used to describe convolution encoders in graphically, they are tree, state and trellis. In this paper we are using the feed forward trellis convolution encoder. Encoded data is transfer to OFDM system. It generates the 100 frames. Each frame is modulated by 16-QAM adding the cyclic prefix of 16bits. 4-pilot carriers are inserted in each frame. The total size if each frame is 96-bits. These are transmitted into the AWGN channel. This will aid the noise in the range of $(0-20)$ dB. Fig.2 shows the CCOFDM is transmitted through an AWGN channel, in this * symbols are representing the noise add the signal and - represents original signal. At the receiving end extract the information bits by viterbi decoding.

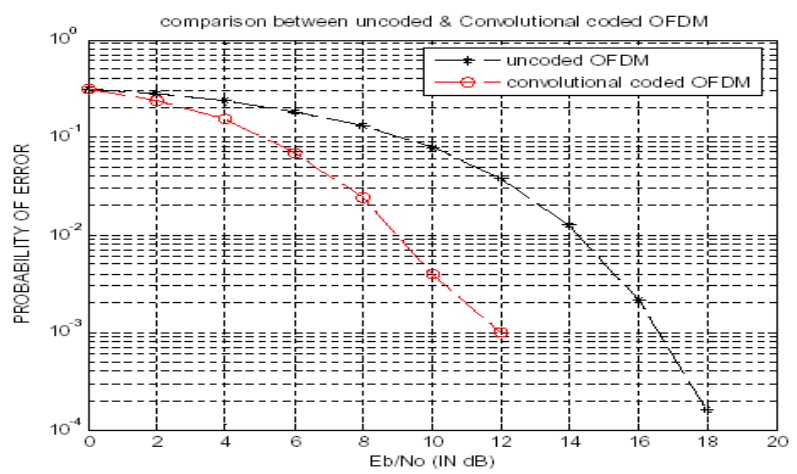

Figure 3. Performance analysis between uncoded and convolution coded OFDM system

According to the study [15] it is known that bursty errors can deteriorate the performance of any communication system. The burst errors can happen either by deep frequency fades or by impulsive noise. Power line channel suffers from both of these deficiencies. To improve the performance of OFDM system we can use FEC code. convolution code is good example of FEC code this result is shown below Fig.3 Convolution coding in OFDM can give performance improvement of some $4 \mathrm{db}$ on AWGN channel over the uncoded OFDM system at required BER .figure 1 shows convolutional codes with QPSK modulation give performance improvement of some $8 \mathrm{~dB}$ over AWGN 
channel gain at $10^{-2}$ for uncoded ofdm is $8 \mathrm{~dB}$ and convolutional coded OFDM is $4.8 \mathrm{~dB}$.

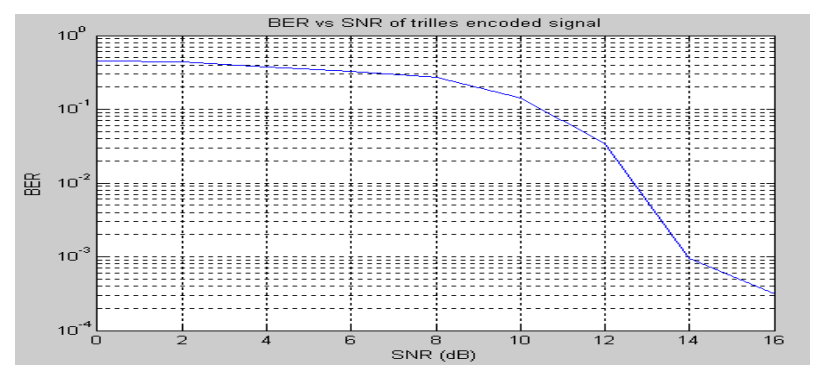

Figure 4. performance of CCOFDM over AWGN channel.

The Fig. 4 the show the convolution coded OFDM system with total number frames are 100 each frame is generated by modulating 16-QAM, FFT size using 64FFTand convolution code rate $1 / 2$.each frame consists of 96-bits and 4 pilots are used the OFDM requires SNR of $8 \mathrm{~dB}$ to achieve a BER of 10-2, SNR of $10 \mathrm{db}$ to achieve a BER o 10-3 so by adding convolution code, the CCOFDM improves the system performance by 4dB.A comparison of uncoded and coded OFDM performance can be improved by $4 \mathrm{~dB}$. But coded OFDM requires memory .so that system complexity and computation is increases.

\section{CONCLUSIONS}

The performance analysis of the convolutional coded OFDM system is evaluated by simulations in AWGN channel. The advantages of using convolutional codes and OFDM are studied separately. It is shown that binary convolutional coded -OFDM can provide a better performance than single carrier Binary convolutional coded ofdm system in fading channels. On the other hand, convolutional codes can eliminate the residual inter symbol interference (ISI) and inter channel interference (ICI) and therefore reduce the length of the required Cyclic prefix in an OFDM system. This decreases the overhead associated with the Cyclic Prefix. The use of convolutional codes in OFDM system for high data rate transmission in wireless LANs, results in a considerable improvement in terms of bit error rate performance and bandwidth efficiency.

\section{REFERENCES}

[1] B.Balaji Naik - Performance Of Turbo Coded OFDM In Wireless Application\| in partial fulfillment of the requirements for the award of Master of Technology degree in Electronics and Communication Engineering with specialization in -VLSI Design \& Embedded system\| during session 2007-2008 at National Institute Of Technology, Rourkela (Deemed University).
[2] I Gede Puja Astawa, Yoedy Moegiharto, Ahmad Zainudin, Imam Dui Agus Salim, Nur Annisa AnggraeniPerformance Analysis of MIMO-OFDM Using Convolution Codes with QAM Modulation in- International Journal of Electrical, Electronic Science and Engineering Vol:7 No:12, 2013 .

[3] Xu Zhang-QAM-based COFDM System in partial fulfillment of the requirements for the award of Master of Technology degree in Master of Science Degree in Electrical Engineering at University of Toledo in August 2011.

[4] Error Control Coding (2nd Edition) [Hardcover] by $\underline{\text { Shu Lin }}$ , Daniel J. Costell.

[5] Analog and Digital communication by sham shanmugam.

[6] contempory communication systems by - proiakis.

[7] Payaswini Pand Manjaiah D.H -ANALYSIS OF EFFECT OF CYCLIC PREFIX ON DATA RATES IN OFDM MODULATION TECHNIQUES International Journal of Advanced Computer and Mathematical Sciences ISSN 2230-9624. Vol 3, Issue 4, 2012.

[8] J.Faezah, and K.Sabira, "Adaptive Modulation for OFDM Systems" International Journal of Communication $\mathrm{Ne}$ tworks and Information Security (IJCNIS) Vol. 1, No. 2, August 2009 .

[9] Peters, S.W., Heath, R.W. "The future of WiMAX:Multihop relaying with IEEE 802.16j" IEEE Communication magazine vol. 47 issue 1, pp. 104-111. Jan 2009

[10] P. Van Eetvelt, G. Wade, and M. Tomlinson, "Peak to average power reduction for OFDM schemes by selective scrambling," Elect. Letter, October 1996, pp. 1963-1964.

[11] Ramjee Prasad, "OFDM for wireless communications systems" (2004), Artech House Inc. ISBN 1-58053-796-0

[12] T.Tsugi, M. Itami, "A study on adaptive modulation of OFDM under impulsive power line channel," IEEE International Symposium on Power Line Communications and Its Applications, ISPLC, pp. 304-309, 2-4 April 2008

[13] U. K. Kwon, G. H. Im, and E. S. Kim, "An iteration technique for recovering insufficient cyclic prefix and clipped OFDM signals," IEEE Signal Process. vol. 14, no. 5, pp. 317- 320, May 2007.

[14] Werner Henkel, George Taubock, Per Odling,Per Ola Borjesson, Niklas Petersson, Albin Johansson, "The Cyclic Prefix of OFDM/DMT - An Analysis", International Zurich Seminar on Broadband Communications access transmission - Networking.

[15] Ms Neetu Sharma, Prof. Rajeshwar Lal Dua-" To improve Bit error Rate OFDM Transmission Using turbo codes."International Journal of Advanced Research in Computer Engineering \& Technology Volume 1, Issue 4, June 2012.

[16] Block Turbo Code And Its Application to OFDM For Wireless Local Area Network\| Phd thesis submitted by Hrudananda Pradhan.

[17] C. Berrou, A. Glavieux, and P. Thitimajshima, -Near Shannon Limit Error-Correcting Coding: Turbo Codes\|, Proceedings of the IEEE International Conference on Communications, ICC ‘93, Geneva., pp. 1064-1070, May 1993.

[18] D. Rajaveerappa, Abdelsalam Almarimi - RSA/Shift Secured IFFT/FFT Based OFDM Wireless System|| in 2009 Fifth International Conference on Information Assurance and

Security. 